\title{
Fluorescence real-time kinetics of protoporphyrin IX after 5-ALA administration in low-grade glioma
}

\author{
*Sadahiro Kaneko, MD,, ${ }^{1,2}$ Eric Suero Molina, Dr med, MBA, ${ }^{1}$ Peter Sporns, Dr med, MHBA, ,,4 \\ Stephanie Schipmann, Dr med, ${ }^{1}$ David Black, ${ }^{5,6}$ and Walter Stummer, Dr med ${ }^{1}$

\begin{abstract}
1Department of Neurosurgery, University Hospital of Münster, Germany; ${ }^{2}$ Department of Neurosurgery, Hokkaido University Graduate School of Medicine, Sapporo, Japan; ${ }^{3}$ Department of Neuroradiology, Clinic of Radiology and Nuclear Medicine, University Hospital Basel, Switzerland; ${ }^{4}$ Department of Diagnostic and Interventional Neuroradiology, University Medical Center Hamburg-Eppendorf, Hamburg, Germany; ${ }^{5} \mathrm{Carl}$ Zeiss Meditec AG, Oberkochen, Germany; and ${ }^{6}$ University of British Columbia, Vancouver, British Columbia, Canada
\end{abstract}

\begin{abstract}
OBJECTIVE 5-Aminolevulinic acid (5-ALA) induces fluorescence in high-grade glioma (HGG), which is used for resection. However, the value of 5-ALA-induced fluorescence in low-grade glioma (LGG) is unclear. Time dependency and time kinetics have not yet been investigated. The purpose of this study was to investigate real-time kinetics of protoporphyrin IX (PpIX) in LGG based on hyperspectral fluorescence-based measurements and identify factors that predict fluorescence.

METHODS Patients with grade II gliomas and imaging from which HGGs could not be completely ruled out received $5-\mathrm{ALA}$ at $20 \mathrm{mg} / \mathrm{kg}$ body weight 4 hours prior to surgery. Fluorescence intensity (FI) and PpIX concentration (CPpIX) were measured in tumor tissue utilizing a hyperspectral camera. Apparent diffusion coefficient (ADC)-based tumor cell density, Ki-67/MIB-1 index, chromosomal 1p/19q codeletion, and ${ }^{18} \mathrm{~F}$-fluoroethyl-L-tyrosine ( $\left.{ }^{18} \mathrm{~F}-\mathrm{FET}\right) \mathrm{PET}$ values and their role for predicting fluorescence were evaluated.
\end{abstract}

RESULTS Eighty-one biopsies from 25 patients were included. Tissues with fluorescence demonstrated FI and CPpIX maxima between 7 and 8 hours after administration. When visible fluorescence was observed, peaks of FI and CPpIX were observed within this 7- to 8-hour time frame, regardless of any MRI gadolinium contrast enhancement. Gadolinium enhancement ( $p=0.008)$, Ki-67/MIB-1 index ( $p<0.001)$, ${ }^{18} \mathrm{~F}-\mathrm{FET}$ PET uptake ratio $(p=0.004)$, and ADC-based tumor cellularity $(p=0.017)$ significantly differed between fluorescing and nonfluorescing tissue, but not $1 p / 19 q$ codeletions. Logistic regression demonstrated that ${ }^{18} \mathrm{~F}-\mathrm{FET}$ PET uptake and Ki-67/MIB-1 index were independently related to fluorescence.

CONCLUSIONS This study reports a fluorescence-based assessment of CPpIX in human LGG tissues related to ${ }^{18} \mathrm{~F}-\mathrm{FET}$ PET uptake and Ki-67/MIB-1. As in HGGs, fluorescence in LGGs peaked between 7 and 8 hours after 5-ALA application, which has consequences for the timing of administration.

https://thejns.org/doi/abs/10.3171/2020.10.JNS202881

KEYWORDS 5-ALA; fluorescence-guided resection; low-grade glioma; PpIX concentration; oncology

$\mathrm{T}$ HE optimal treatment for low-grade gliomas (LGGs) is a matter of ongoing debate. 5-Aminolevulinic acid (5-ALA) is a broadly used and well-established surgical adjunct in high-grade glioma (HGG) surgery. The randomized phase III multicenter approval study of 5-ALA is one of the most cited articles in neurosurgery. ${ }^{1}$ This is not only due to the high clinical relevance in glioma surgery, but also due to the variable application of 5-ALA in translational research. However, the clinical value of 5-ALA in LGG surgery remains an ongoing discussion. ${ }^{2,3}$ Specifically, time dependency and time kinetics have not been well investigated, and hypothetically, LGGs with lower growth rates might display different kinetics.

To date, it has been reported that $10 \%-23 \%$ of LGGs will fluoresce. ${ }^{2-5}$ Jaber et al. described tumor, volume, age, and an ${ }^{18} \mathrm{~F}$-fluoroethyl-L-tyrosine $\left({ }^{18} \mathrm{~F}\right.$-FET) PET uptake

ABBREVIATIONS 5-ALA = 5-aminolevulinic acid; ${ }^{18} \mathrm{~F}-\mathrm{FET}={ }^{18} \mathrm{~F}$-fluoroethyl-L-tyrosine; $\mathrm{ADC}=$ apparent diffusion coefficient; $\mathrm{AUC}=$ area under the curve; $\mathrm{BBB}=$ blood-brain barrier; $\mathrm{Cl}=$ confidence interval; $\mathrm{CPpIX}=\mathrm{PpIX}$ concentration; $\mathrm{FI}$ = fluorescence intensity; HGG = high-grade glioma; IDH = isocitrate dehydrogenase; LGG = low-grade glioma; $\mathrm{PpIX}=$ protoporphyrin IX; ROI = region of interest; SUVmax = maximum standardized uptake value.

SUBMITTED July 25, 2020. ACCEPTED October 30, 2020.

INCLUDE WHEN CITING Published online June 18, 2021; DOI: 10.3171/2020.10.JNS202881.

* S.K. and E.S.M. contributed equally to this work. 
ratio greater than 1.85 as predictors for fluorescence in these tumors. ${ }^{3}$ Additionally, any contrast enhancement in MRI further increased the probability of visible fluorescence. Furthermore, fluorescent tumors presented a significantly higher Ki-67/MIB-1 index (i.e., $14.5 \%$ vs 5.8\%) than nonfluorescent LGGs, ${ }^{3}$ indicating high proliferation, which several authors have found to be correlated with visible fluorescence ${ }^{6,7}$ In contrast to HGGs, protoporphyrin IX (PpIX) fluorescence in LGGs might aid in finding an anaplastic focus, which can be found in $44 \%-55 \%$ of tumors with typical LGG MRI aspects. ${ }^{8}{ }^{8}{ }^{18} \mathrm{~F}-\mathrm{FET}$ PET can further serve as an additional tool to search for these anaplastic foci-regions of high proliferation rate-prior to surgery. ${ }^{10}$ This is based on observations of previous authors who have found both heterogenous ${ }^{18} \mathrm{~F}$-FET PET uptake in $\mathrm{LGG}^{8}$ and a correlation of these regions with visible fluorescence. ${ }^{6,7} \mathrm{With}$ the help of fluorescence, these regions can be precisely targeted to ensure correct histopathological diagnosis and avoid undergrading of pathological tissue to confirm the true tumor grade, ${ }^{3,4}$ which is relevant for adjuvant treatment and prognosis. Although preoperative imaging might lead the way for finding these anaplastic foci, intraoperatively, brain shift may lower the accuracy of conventional image guidance.

To our knowledge, reports regarding the kinetics and metabolism of 5-ALA in LGG-e.g., how and when the maximum concentration of PpIX peak after 5-ALA administration can be found-are missing from the present literature. In a recent article, we evaluated the real-time kinetics of PpIX in HGG based on fluorescence measurements with a hyperspectral camera. ${ }^{11}$ We demonstrated that the PpIX concentration (CPpIX) peaked later than previously thought in HGG, i.e., 7-8 hours after 5-ALA administration. This was in contrast to data from an early animal experiment published in 1998, in which a fluorescence peak was observed 6 hours after 5-ALA administration, ${ }^{12}$ a regime later adopted not only by the standard product characteristics of Gliolan (Medac), but also by the vast majority of published clinical trials.,.$^{1,313-18}$ These results are consistent with other studies evaluating CPpIX in plasma (7.8 hours) ${ }^{15}$ or skin (6.5-9.8 hours).$^{19}$

Hence, the main objective of this study was to investigate the PpIX real-time kinetics after 5-ALA administration in a subset of patients with glioma found to harbor LGG as a final pathological diagnosis, to determine the best timing for surgery when utilizing 5-ALA-mediated fluorescence-guided resection. Furthermore, we analyzed different factors that predict fluorescence in this patient collective.

\section{Methods \\ Patients}

This study evaluated adult patients with LGG who were surgically treated at the Department of Neurosurgery, University Hospital of Münster, between January and December 2018. These patients were selected for dosing with 5-ALA based on large tumor size, old age, any degree of spurious enhancement, an elevated ${ }^{18}$ F-FET signal on PET, or other factors that increased the likelihood of the tumor harboring areas of malignant degeneration. ${ }^{3}$ Ulti- mately, these tumors were histopathologically confirmed as low grade, despite biopsy specimens being specifically collected from fluorescent regions in cases with fluorescence. All surgeries, as well as the intraoperative fluorescence assessment, were performed by two senior surgeons (W.S. and E.S.M.).

5-ALA (Gliolan, medac $\mathrm{GmbH}$ ) was administered at a standard dose of $20 \mathrm{mg} / \mathrm{kg}$ body weight 4 hours prior to induction of anesthesia. The timing of administration was recorded for each patient. To visualize fluorescence during surgery, the surgical microscope OPMI Pentero 900 (Carl Zeiss AG) was used. Visible fluorescence quality was assessed by the operating surgeon. We collected and analyzed tumor tissue, regardless of visible fluorescence. When feasible, we collected tissue at a minimum of three different time points during surgery. Tissues were subsequently submitted for neuropathological analyses.

Each patient's written informed consent was obtained. All procedures performed in studies involving human participants were in accordance with the ethical standards of the institutional research committee and with the 1964 Helsinki Declaration and its later amendments or comparable ethical standards. Ex vivo tissue evaluation was approved by the local ethics committee of the University of Münster.

MRI examinations were acquired within 2 weeks prior to surgery. Enhancement on T1-weighted images after gadolinium-based contrast agent administration was categorized into two groups, i.e., positive or negative, as previously described., ${ }^{9,20}$ Because the apparent diffusion coefficient (ADC) has been reported to reflect tumor cellularity, we analyzed available imaging in our patient collective according to the method published by Hayashida et al. ${ }^{21}{ }^{18}$ F-FET PET uptake (maximum standardized uptake value [SUVmax]) was obtained for comparison. We also stratified ratios into two groups, i.e., smaller or larger than 1.85 , as previously described. ${ }^{3}$

\section{Spectrometric Measurement}

Spectrometric measurements were performed using a hyperspectral imaging system in 10 regions of interest (ROIs) per tumor sample to obtain a fluorescence intensity (FI) as previously described. ${ }^{11}$ Tissues were measured ex vivo directly after collection, paying careful attention to light protection from light exposure during transfer to the imaging system. White light and pure fluorescence spectra of the ROI were captured. Using normalization factors obtained from the white light intensity in certain spectral regions as well as an empirically derived exponent, the fluorescence spectra were normalized for inhomogeneous scattering and absorption properties across the tissue. The relative PpIX FI was then derived by isolating the PpIX spectrum from the remaining signal by taking the least squares solution to an overdetermined linear system of equations. This analysis was conducted using commercially available software (MATLAB, MathWorks Inc.). Furthermore, our algorithm was calibrated using fluorescence phantoms with known CPpIX. ${ }^{11}$ This provided the CPpIX values used here. It is important to note that these values are precise and self-consistent within this study, so the relative differences in CPpIX upon which this study 
relies are exact and reliable. However, accurate comparison of absolute CPpIX values with measurements from other groups with other devices was not intended.

\section{Histology}

All tumor tissues, including the biopsy specimens used for ex vivo spectroscopy, were assessed histologically according to the WHO classification of 2016. ${ }^{22}$ Isocitrate dehydrogenase-1 (IDH-1) mutation status was determined using immunohistochemistry or using multiplex ligationdependent probe amplification analysis, if needed. ${ }^{23}$

\section{Statistical Analysis}

For statistical purposes, we categorized the time after administration into four groups of 1-hour intervals each. Distribution was evaluated with the F-test between two groups and Bartlett's test for the average among elapsed time groups. For parametric testing, differences between two groups were investigated using Fisher's exact test for categorical data; for nonparametric testing, the KruskalWallis test was used; and to adjust for multiple comparisons, the Bonferroni method was used. Multivariate analyses were performed using a logistic regression model and were characterized by $95 \%$ confidence intervals (CIs). Differences were regarded as statistically significant if the error probability $\mathrm{p}$ was less than $5 \%$. All statistical analyses were performed with either IBM SPSS statistical software (version 25.0, IBM Corp.) or EZR (Saitama Medical Center, Jichi Medical University), which is a graphical user interface of the R Foundation for Statistical Computing. ${ }^{24}$ To assess the prediction of fluorescence, receiver operating characteristic curves were conducted, with an area under the curve (AUC) $\geq 0.7$ being assumed to demonstrate reasonable discrimination.

\section{Results}

\section{Patients and Tissues}

Patient demographics are shown in Table 1. This study included 25 patients (11 men and 14 women, mean age 43 years). Fifteen patients (60\%) were diagnosed as having diffuse astrocytoma (IDH-mutant), $2(8 \%)$ as diffuse astrocytoma (IDH-wild type), and 8 (32\%) as oligodendroglioma (IDH-mutant, $1 \mathrm{p} / 19 \mathrm{q}$ codeleted). All tumors were grade II according to the latest WHO classification. ${ }^{22}$ Intraoperatively, $9(36 \%)$ of 25 patients demonstrated visible fluorescence during surgery (Table 1). A total of 81 tissues were collected for measurement. Visible fluorescence was detected in $21(25.9 \%)$ of 81 tissues.

\section{Time Dependency of FI and CPpIX}

CPpIX and FI were significantly higher in tissues, where visible fluorescence was observed using the microscope intraoperatively ( $<<0.001$; Fig. 1$)$. Figure $2 \mathrm{~A}$ demonstrates CPpIX in fluorescing tissue peaking between 7 and 8 hours after 5-ALA administration. FI was similar, demonstrating its highest level between 7 and 8 hours (Fig. 2B). Nonfluorescing tissues maintained low CPpIX and FI regardless of the time frame, and these values seldom fluctuated.
TABLE 1. Patient demographics

\begin{tabular}{|c|c|}
\hline Variable & No. of Patients (\%) \\
\hline All & $25(100)$ \\
\hline \multicolumn{2}{|l|}{ Sex } \\
\hline Male & $11(44)$ \\
\hline Female & $14(56)$ \\
\hline Mean age \pm SD, yrs & $43 \pm 10.3$ \\
\hline \multicolumn{2}{|l|}{ Histology } \\
\hline Diffuse astrocytoma, IDH-mutant & $15(60)$ \\
\hline Diffuse astrocytoma, IDH-wild type & $2(8)$ \\
\hline $\begin{array}{l}\text { Oligodendroglioma, IDH-mutant \& 1p/19q } \\
\text { codeletion }\end{array}$ & $8(32)$ \\
\hline \multicolumn{2}{|l|}{ KPS score } \\
\hline 80 & $2(8)$ \\
\hline 90 & $7(28)$ \\
\hline 100 & $16(64)$ \\
\hline \multicolumn{2}{|l|}{ MRI enhancement } \\
\hline Positive & $6(24)$ \\
\hline Negative & $19(76)$ \\
\hline \multicolumn{2}{|l|}{ Fluorescence visibility } \\
\hline Positive & $9(36)$ \\
\hline Negative & $16(64)$ \\
\hline
\end{tabular}

KPS = Karnofsky Performance Scale.

\section{Fluorescence Visibility, MRI Enhancement, ${ }^{18}$ F-FET PET Uptake Ratio, and ADC-Based Measured Tumor Cellularity}

Gadolinium enhancement on MRI was significantly correlated with the presence of visible fluorescence $(\mathrm{p}=$ 0.008; Table 2). For both FI and CPpIX, we observed a trend toward higher values but no statistically significant difference between visible fluorescence and existing contrast enhancement ( $p$ value nonsignificant; Fig. 3). ADCbased tumor cellularity correlated with visible fluorescence $(p=0.017)$ in univariate analysis. Time kinetics of CPpIX and FI stratified by MRI enhancement revealed a peak between 7 and 8 hours in both groups (Fig. 3).

Stratifying SUVmax into values below and above 1.85 (as previously described ${ }^{3}$ ) within our fluorescent and nonfluorescent tissues did not demonstrate a significant difference among the evaluated groups ( $p$ value nonsignificant). However, evaluating absolute SUVmax within fluorescing $(\mathrm{n}=54$; median 2.26, IQR 1.59-3.1) and nonfluorescing ( $\mathrm{n}=15$; median 3.86, IQR 1.97-5.7) tissue demonstrated a significant difference between both fluorescing and nonfluorescing tissue ( $p=0.004$; Table 2). Validation of $a b-$ solute values of ${ }^{18} \mathrm{~F}-\mathrm{FET}$ PET uptake (SUVmax) resulted in an AUC of 0.7 when comparing within fluorescing and nonfluorescing tissue, indicating a fair discrimination.

\section{Ki-67/MIB-1 Index and 1p/19q Codeletion}

Measured Ki-67/MIB-1 indices in nonfluorescing (n $=60$, mean 5.4\%) and fluorescing $(\mathrm{n}=21$, mean $8.29 \%)$ tissues differed significantly $(\mathrm{p}<0.001)$. Nonfluorescent samples were negative for a chromosomal $1 \mathrm{p} / 19 \mathrm{q}$ codeletion in 46 cases and positive in 14, whereas fluorescing 


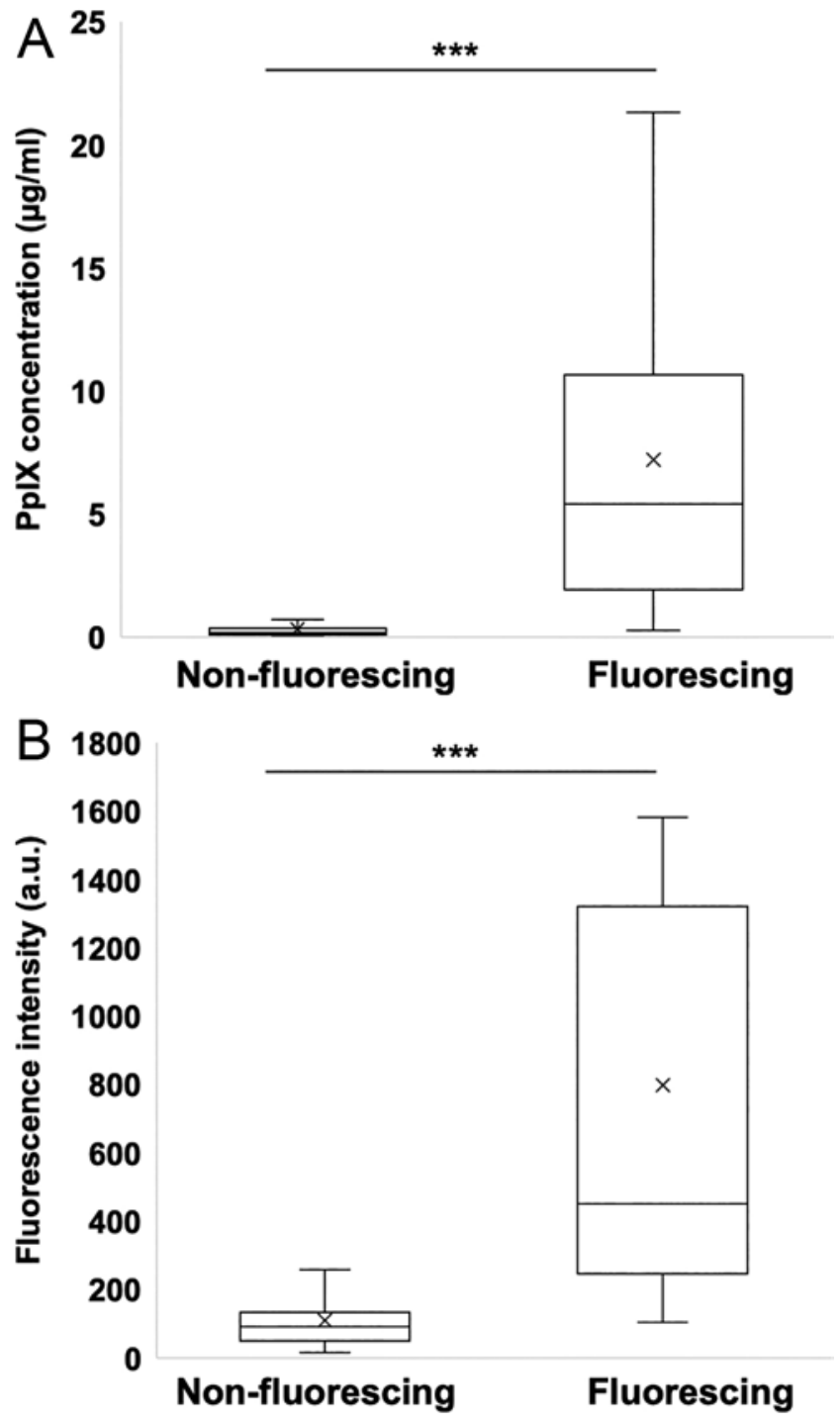

FIG. 1. Box-and-whisker plots demonstrating the relationship between visible fluorescence and CPpIX (A) or FI (B). Visible fluorescence was significantly correlated with CPpIX and $\mathrm{FI}\left({ }^{* * *} \mathrm{p}<0.001\right)$. The mean is represented by " $x$." a.u. = arbitrary units. tissue did not demonstrate a codeletion in 12 cases and was present in 9. Stratifying between positive and negative chromosomal $1 \mathrm{p} / 19 \mathrm{q}$ codeletion among both fluorescing groups did not demonstrate any significant difference.

\section{Multivariate Analysis}

From all analyzed variables, a logistic regression model demonstrated the Ki-67/MIB-1 index ( $\mathrm{p}=0.011$, OR $3.663,95 \%$ CI $1.355-9.904)$ and ${ }^{18}$ F-FET PET absolute SUVmax ( $p=0.039$, OR 5.958, 95\% CI 1.092-32.514) to be independent factors for predicting fluorescence. Gadolinium enhancement on MRI, ADC-based cellularity, and $1 \mathrm{p} / 19 \mathrm{q}$ codeletion did not reach statistical significance in multivariate analysis (Table 2).

\section{Discussion}

The characteristic invasive growth of gliomas represents the biggest challenge when treating this malignancy. Predominantly, LGGs tend to deeply infiltrate adjacent parenchyma. ${ }^{25}$ Early resection has been shown to be beneficial, demonstrating better overall survival in comparison with biopsy and the "wait and see" approach. ${ }^{26}$ Furthermore, increasing the extent of resection with small residual tumor increases both the overall and progression-free survival in these patients. ${ }^{25,27-29}$ Both observations have led to the recommendation for maximal or at least submaximal safe resection as an initial step in the treatment algorithm of these patients. ${ }^{20}$ However, the prognostic value of visible fluorescence in LGGs remains under investigation. In a recent study, Jaber et al. demonstrated a significantly shorter progression-free (median 9.8 vs 45.8 months), malignant transformation-free (median 43 vs 64.6 months), and overall (51.6 vs 68.2 months) survival in fluorescent, histologically confirmed LGGs compared with those without any visible fluorescence, despite similar low MIB indices. ${ }^{2}$ Hence, fluorescence in LGG predicted a worse outcome in these patients.

Fluorescence-guided resection with 5-ALA has become a standard surgical adjunct when resecting HGG, now implemented worldwide. ${ }^{1}$ Even though it has been broadly applied for more than a decade, its thorough me-
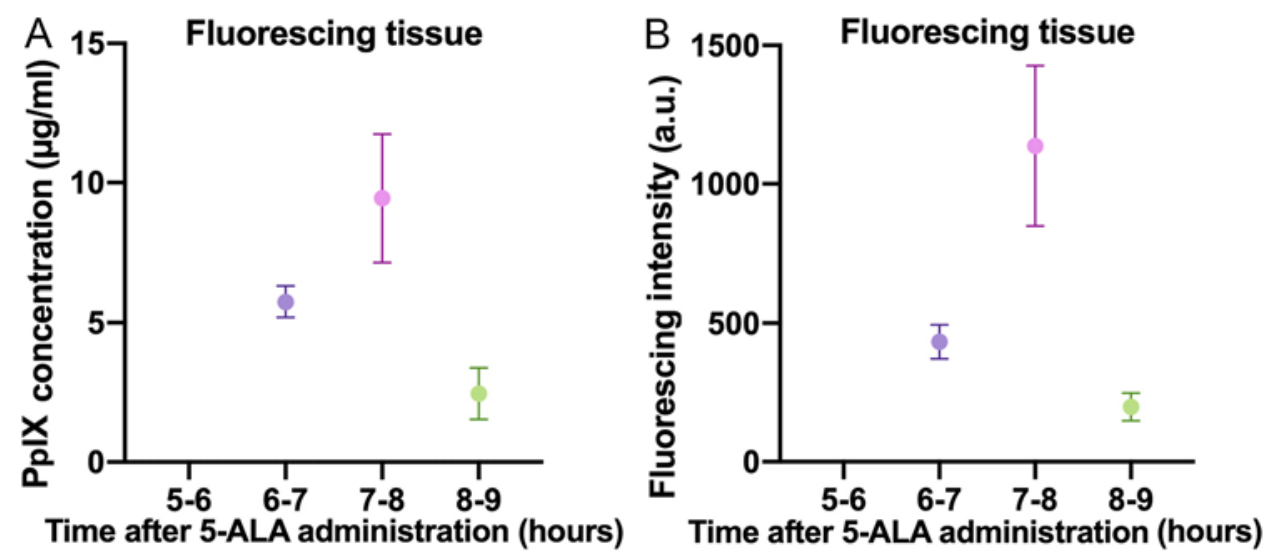

FIG. 2. Time dependency of $\mathrm{CPpIX}$ and FI categorized by intraoperative visible fluorescence. In fluorescent tissue, the maximum average of both CPpIX (A) and FI (B) was observed 7-8 hours after 5-ALA administration. Nonfluorescent tissue (not displayed) did not demonstrate a relevant time kinetic. The error bar represents the standard error of the mean. Figure is available in color online only. 
TABLE 2. Univariate and multivariate analyses of different variables among fluorescing and nonfluorescing tissue and their role for predicting fluorescence

\begin{tabular}{lrccc}
\hline & Univariate & \multicolumn{3}{c}{ Multivariate Analysis } \\
\cline { 3 - 5 } Variable & Analysis & $95 \% \mathrm{Cl}$ & $\mathrm{p}$ Value & OR \\
\hline Gd enhancement on MRI (yes/no) & $\mathbf{0 . 0 0 8}$ & $0.004-2.161$ & 0.138 & 0.09 \\
\hline MIB-1 index (\%) & $<0.001$ & $1.355-9.904$ & $\mathbf{0 . 0 1 1}$ & 3.663 \\
\hline${ }^{18}$ F-FET PET SUVmax (absolute value) & $\mathbf{0 . 0 0 4}$ & $1.092-32.514$ & $\mathbf{0 . 0 3 9}$ & 5.958 \\
\hline 1p/19q codeletion (yes/no) & 0.090 & $0.043-10.537$ & 0.766 & 0.671 \\
\hline ADC-based cellularity (absolute value) & $\mathbf{0 . 0 1 7}$ & $0-1.719$ & 0.081 & 0.008 \\
\hline
\end{tabular}

Boldface type indicates statistical significance.

tabolization for preferential accumulation of PpIX in human tumor tissue has not yet been fully understood. In the present study, we performed a quantification of visible FI over time and additionally analyzed a time-based dynamic of PpIX in LGG tissue. Time kinetics of fluorescence in this ex vivo study revealed that the highest FI and CPpIX in LGG were observed 7-8 hours after 5-ALA administration, similar to that demonstrated in HGG, where fluorescence likewise peaked after $7-8$ hours ${ }^{11}$ (Fig. 2). Previous published data of PpIX evaluation revealed a peak in plasma at 7.8 hours, ${ }^{15}$ the results of this study are in accordance with those results. These results suggest that tumor cells in LGG follow the same kinetics as in $\mathrm{HGG}^{11}$ counterparts, despite a lower proliferation rate.

In addition, our findings have implications for the timing of surgery after 5-ALA medication. Present recommendations outlined in the standard product characteristics for 5-ALA favor beginning medication 2.5-3.5 hours prior to surgery. We recommend dosing 4-5 hours prior to induction of anesthesia, adapted to a center's logistics, such as the average time to reach the tumor of each institution. Moreover, optimizing surgery according to the time dependency of this fluorophore might improve fluorescence visualization, especially in weakly fluorescing tumors. We observed visible fluorescence in $39 \%$ of evalu- ated patients, which is higher than in previous reports ${ }^{2-5}$ but may be related to our case selection of larger tumors, tumors with a positive ${ }^{18} \mathrm{~F}-\mathrm{FET}$ signal, or any enhancement.

Early angiogenic changes, such as increased permeability, intravascular volume, or tumor vessel density, have been proposed as explanations for the accumulation of PpIX in LGGs. ${ }^{2,30,31}$ 5-ALA alone does not cross the intact blood-brain barrier (BBB). ${ }^{31}$ However, increases in BBB permeability lead to enhancement on MRI after gadolinium administration and to higher ${ }^{18} \mathrm{~F}$-FET uptake on PET. ${ }^{32}$ We observed a significant difference in CPpIX depending on the presence or absence of contrast enhancement on MRI ( $\mathrm{p}=0.008$; Table 2). Interestingly, FI and CPpIX were also observed in nonenhancing tumors, with similar FI and PpIX time kinetics as compared to enhancing tumors (Fig. 3). Thus, even in LGG without MRI contrast enhancement, which demonstrates visible fluorescence in $11 \%-13 \%$ of cases, ${ }^{3,4}$ a similar time dependency of 5-ALA was observed (Fig. 3). We detected higher ${ }^{18} \mathrm{~F}-$ FET PET uptake values with increasing CPpIX, the difference reaching statistical significance in both univariate analysis $(\mathrm{p}=0.004)$ and multivariate logistic regression $(p=0.039$, OR 5.958, 95\% CI 1.092-32.514). Contrary to earlier studies, ${ }^{2,3,6}$ we did not observe a significant correla-
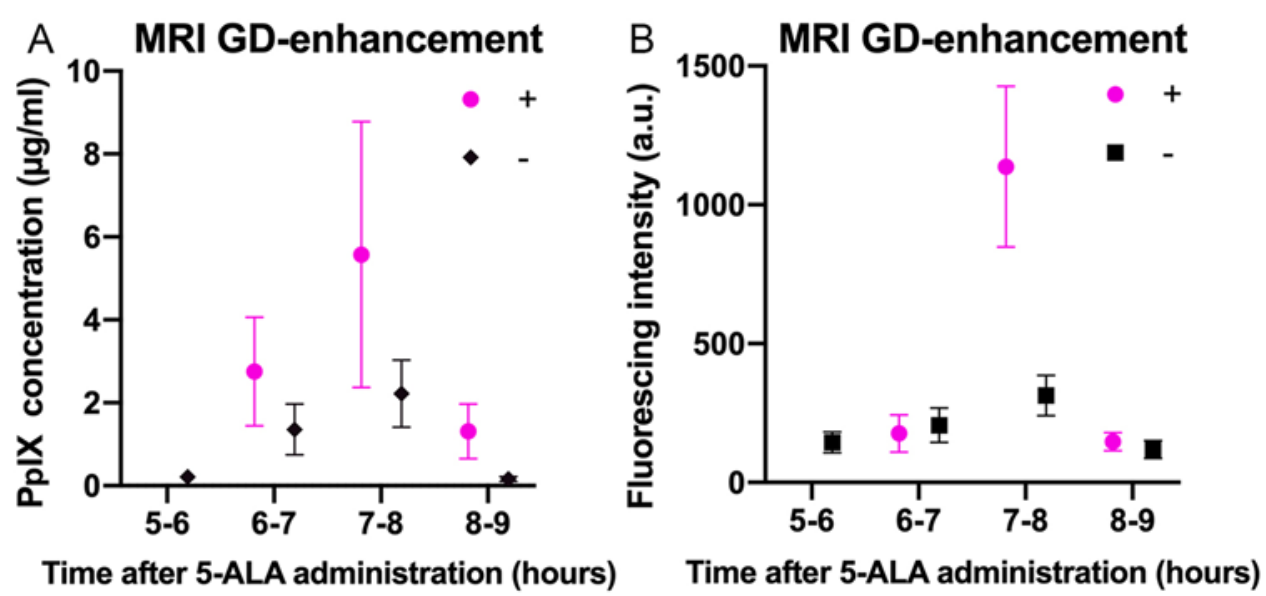

FIG. 3. CPpIX (A) and FI (B) in tumor tissue categorized by MRI enhancement. If tumors demonstrated positive MRI enhancement prior to surgery, tissues tended to have higher values of both CPpIX and FI than negative MRI enhancement. For both groups, values peaked 7-8 hours after 5-ALA medication. The error bar represents the standard error of the mean. Figure is available in color online only. 


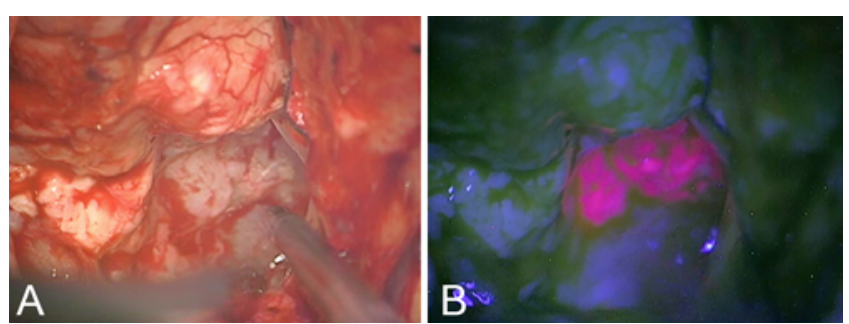

FIG. 4. Intraoperative microscope imaging during fluorescence-guided resection of a left frontal IDH-1-mutated astrocytoma using conventional white light (A) and a BLUE 400 filter (B). Figure is available in color online only.

tion between ${ }^{18} \mathrm{~F}$-FET PET uptake ratios below and above 1.85 and visible fluorescence. This might be explained by the number of analyzed patients in this study. However, absolute SUVmax still differed significantly between fluorescing and nonfluorescing biopsies $(p=0.004$; Table 2), underscoring the clinical value of PET diagnostics in predicting fluorescence and in aiding surgeons in implementing fluorescence guidance in these patients. Furthermore, the AUC of ${ }^{18} \mathrm{~F}-\mathrm{FET}$ PET uptake (SUVmax) was 0.7, indicating satisfactory predictability for the presence of fluorescence.

A further interesting finding was the role of the Ki-67/ MIB-1 labeling index in predicting fluorescence. Ki-67 is associated with proliferation and aids in grading malignancy in gliomas. ${ }^{22}$ In both univariate $(\mathrm{p}<0.001)$ and multivariate ( $\mathrm{p}=0.038$, OR $3.663,95 \%$ CI $1.059-7.396)$ analyses, the Ki-67/MIB-1 index was significantly higher in fluorescing tissue, indicating an LGG that harbored fluorescence to demonstrate higher proliferation ratios. The ADC-based cellularity measurement likewise presented higher values in fluorescent tumors in univariate analysis $(\mathrm{p}=0.017)$. Both of these parameters can indicate higher malignancy in these tumors. Similarly, the presence of fluorescence has been associated with worse prognosis in $\mathrm{LGG}^{2}$ (Fig. 4).

As previously reported, ${ }^{11}$ we observed no disparities between the measured FI and calculated CPpIX in our patient collective. Our group has stressed the importance of time dependency in the past. For fluorophores with both intravenous applications, which requires a disruption of the BBB (e.g., fluorescein sodium), ${ }^{33}$ and 5-ALA-induced porphyrin fluorescence in the context of $\mathrm{HGG},{ }^{11}$ time dependency of fluorescence is a factor that plays a role for practical use or experimental evaluations. It is therefore important to understand this factor and adapt the administration of dyes accordingly.

Based on our clinical and published experience, ${ }^{2,3}$ an indication for the use of fluorescence guidance for resection of gliomas without typical glioblastoma imaging features should be sought according to age ( $>44$ years of age), tumor volume $\left(>6.7 \mathrm{~cm}^{3}\right)$, any type of contrast enhancement on MRI, and ${ }^{18}$ F-FET PET uptake (uptake ratio $>1.85$ ) in these patients.

\section{Conclusions}

Our analysis of 81 tissue samples from 25 patients with LGG and measurement of CPpIX and FI demonstrated a fluorescence peak approximately 7-8 hours after 5-ALA administration. Furthermore, ${ }^{18} \mathrm{~F}-\mathrm{FET}$ PET uptake and Ki-67/MIB-1 were independent strong factors for predicting fluorescence. Particularly in these tumors, the timing of 5-ALA medication has to be adapted to each individual institution's surgical workflow for optimizing visualization of PpIX-mediated fluorescence, given their frequently weaker fluorescence.

\section{References}

1. Stummer W, Pichlmeier U, Meinel T, et al. Fluorescenceguided surgery with 5-aminolevulinic acid for resection of malignant glioma: a randomised controlled multicentre phase III trial. Lancet Oncol. 2006;7(5):392-401.

2. Jaber M, Ewelt C, Wolfer J, et al. Is visible aminolevulinic acid-induced fluorescence an independent biomarker for prognosis in histologically confirmed (World Health Organization 2016) low-grade gliomas? Neurosurgery. 2019;84(6): $1214-1224$.

3. Jaber M, Wölfer J, Ewelt C, et al. The value of 5-aminolevulinic acid in low-grade gliomas and high-grade gliomas lacking glioblastoma imaging features: an analysis based on fluorescence, magnetic resonance imaging, $18 \mathrm{~F}$-fluoroethyl tyrosine positron emission tomography, and tumor molecular factors. Neurosurgery. 2016;78(3):401-411.

4. Widhalm G, Kiesel B, Woehrer A, et al. 5-Aminolevulinic acid induced fluorescence is a powerful intraoperative marker for precise histopathological grading of gliomas with nonsignificant contrast-enhancement. PLoS One. 2013;8(10): e76988.

5. Nishikawa R. Fluorescence illuminates the way.... Neuro Oncol. 2011;13(8):805.

6. Ewelt C, Floeth FW, Felsberg J, et al. Finding the anaplastic focus in diffuse gliomas: the value of Gd-DTPA enhanced MRI, FET-PET, and intraoperative, ALA-derived tissue fluorescence. Clin Neurol Neurosurg. 2011;113(7):541-547.

7. Stockhammer F, Misch M, Horn P, et al. Association of F18fluoro-ethyl-tyrosin uptake and 5-aminolevulinic acid-induced fluorescence in gliomas. Acta Neurochir (Wien). 2009; 151(11):1377-1383.

8. Kunz M, Thon N, Eigenbrod S, et al. Hot spots in dynamic ${ }^{18}$ FET-PET delineate malignant tumor parts within suspected WHO grade II gliomas. Neuro Oncol. 2011;13(3):307-316.

9. Stockhammer F, Plotkin M, Amthauer H, et al. Correlation of F-18-fluoro-ethyl-tyrosin uptake with vascular and cell density in non-contrast-enhancing gliomas. J Neurooncol. 2008; 88(2):205-210.

10. Hollon T, Stummer W, Orringer D, Suero Molina E. Surgical adjuncts to increase the extent of resection: intraoperative MRI, fluorescence, and raman histology. Neurosurg Clin $N$ Am. 2019;30(1):65-74.

11. Kaneko S, Suero Molina E, Ewelt C, et al. Fluorescencebased measurement of real-time kinetics of protoporphyrin IX after 5-aminolevulinic acid administration in human in situ malignant gliomas. Neurosurgery. 2019;85(4):E739E746.

12. Stummer W, Stocker S, Novotny A, et al. In vitro and in vivo porphyrin accumulation by $\mathrm{C} 6$ glioma cells after exposure to 5-aminolevulinic acid. J Photochem Photobiol B. 1998;45(23):160-169.

13. Pichlmeier U, Bink A, Schackert G, Stummer W. Resection and survival in glioblastoma multiforme: an RTOG recursive partitioning analysis of ALA study patients. Neuro Oncol. 2008;10(6):1025-1034.

14. Stummer W, Reulen HJ, Meinel T, et al. Extent of resection and survival in glioblastoma multiforme: identification of and adjustment for bias. Neurosurgery. 2008;62(3):564-576. 
15. Stummer W, Stepp H, Wiestler OD, Pichlmeier U. Randomized, prospective double-blinded study comparing 3 different doses of 5-aminolevulinic acid for fluorescence-guided resections of malignant gliomas. Neurosurgery. 2017;81(2): 230-239.

16. Stummer W, Tonn JC, Goetz C, et al. 5-Aminolevulinic acid-derived tumor fluorescence: the diagnostic accuracy of visible fluorescence qualities as corroborated by spectrometry and histology and postoperative imaging. Neurosurgery. 2014;74(3):310-320.

17. Stummer W, Tonn JC, Mehdorn HM, et al. Counterbalancing risks and gains from extended resections in malignant glioma surgery: a supplemental analysis from the randomized 5-aminolevulinic acid glioma resection study. Clinical article. $J$ Neurosurg. 2011;114(3):613-623.

18. Lau D, Hervey-Jumper SL, Chang S, et al. A prospective Phase II clinical trial of 5-aminolevulinic acid to assess the correlation of intraoperative fluorescence intensity and degree of histologic cellularity during resection of high-grade gliomas. J Neurosurg. 2016;124(5):1300-1309.

19. Rick K, Sroka R, Stepp H, et al. Pharmacokinetics of 5-aminolevulinic acid-induced protoporphyrin IX in skin and blood. J Photochem Photobiol B. 1997;40(3):313-319.

20. Soffietti R, Baumert BG, Bello L, et al. Guidelines on management of low-grade gliomas: report of an EFNS-EANO Task Force. Eur J Neurol. 2010;17(9):1124-1133.

21. Hayashida Y, Hirai T, Morishita S, et al. Diffusion-weighted imaging of metastatic brain tumors: comparison with histologic type and tumor cellularity. AJNR Am J Neuroradiol. 2006;27(7):1419-1425.

22. Louis DN, Perry A, Reifenberger G, et al. The 2016 World Health Organization Classification of Tumors of the Central Nervous System: a summary. Acta Neuropathol. 2016;131(6): 803-820.

23. Capper D, Zentgraf H, Balss J, et al. Monoclonal antibody specific for IDH1 R132H mutation. Acta Neuropathol. 2009; 118(5):599-601.

24. Kanda Y. Investigation of the freely available easy-to-use software 'EZR' for medical statistics. Bone Marrow Transplant. 2013;48(3):452-458.

25. Hervey-Jumper SL, Berger MS. Evidence for improving outcome through extent of resection. Neurosurg Clin N Am. 2019;30(1):85-93.

26. Jakola AS, Myrmel KS, Kloster R, et al. Comparison of a strategy favoring early surgical resection vs a strategy favoring watchful waiting in low-grade gliomas. JAMA. 2012; 308(18):1881-1888.

27. Smith JS, Chang EF, Lamborn KR, et al. Role of extent of resection in the long-term outcome of low-grade hemispheric gliomas. J Clin Oncol. 2008;26(8):1338-1345.

28. McGirt MJ, Chaichana KL, Attenello FJ, et al. Extent of surgical resection is independently associated with survival in patients with hemispheric infiltrating low-grade gliomas. Neurosurgery. 2008;63(4):700-708.
29. Coburger J, Merkel A, Scherer M, et al. Low-grade glioma surgery in intraoperative magnetic resonance imaging: results of a multicenter retrospective assessment of the German Study Group for Intraoperative Magnetic Resonance Imaging. Neurosurgery. 2016;78(6):775-786.

30. Novotny A, Stummer W. 5-Aminolevulinic acid and the blood-brain barrier-a review. Med Laser Appl. 2003;18(1): $36-40$.

31. Ennis SR, Novotny A, Xiang J, et al. Transport of 5-aminolevulinic acid between blood and brain. Brain Res. 2003; 959(2):226-234.

32. Runge VM, Clanton JA, Price AC, et al. The use of Gd DTPA as a perfusion agent and marker of blood-brain barrier disruption. Magn Reson Imaging. 1985;3(1):43-55.

33. Suero Molina E, Stummer W. Where and when to cut? Fluorescein guidance for brain stem and spinal cord tumor surgery-technical note. Oper Neurosurg (Hagerstown). 2018;15(3):325-331.

\section{Disclosures}

Walter Stummer has received speaker and consultant fees from Medac, Zeiss, Leica, Photonamic, and NXDC, and research support from Zeiss. Carl Zeiss Meditec (Oberkochen, Germany) provided the authors with the required BLUE 400 filter and the OPMI PICO system.

\section{Author Contributions}

Conception and design: Suero Molina, Black, Stummer. Acquisition of data: Suero Molina, Kaneko, Sporns. Analysis and interpretation of data: Suero Molina, Schipmann, Black, Stummer. Drafting the article: Suero Molina, Stummer. Critically revising the article: all authors. Reviewed submitted version of manuscript: all authors. Approved the final version of the manuscript on behalf of all authors: Suero Molina. Statistical analysis: Suero Molina, Kaneko, Schipmann, Stummer. Administrative/technical/ material support: Suero Molina, Kaneko, Black, Stummer. Study supervision: Suero Molina, Stummer.

\section{Supplemental Information \\ Previous Presentations}

Portions of this manuscript were published as an e-poster at the 2020 virtual AANS Annual Meeting.

\section{Correspondence}

Eric Suero Molina: University Hospital of Münster, Germany. eric.suero@ukmuenster.de. 